\title{
UN MODELO DINÁMICO PARA REDUCIR LA CONTAMINACIÓN EN MÉXICO
}

\author{
A DYNAMIC MODEL FOR REDUCING POLLUTION \\ IN MEXICO \\ JUAN C. CASTRO-RAMÍREZ* \\ Miguel A. GutiérRez-Andrade ${ }^{\dagger}$ Elsa P. OMAÑA-Pulido \\ SERGIO G. DE-LOS-COBOS-SILVA ${ }^{\S}$
}

Received: 9/May/2018; Revised: 9/May/2019;

Accepted: 23/May/2019

\begin{abstract}
Revista de Matemática: Teoría y Aplicaciones is licensed under a Creative Commons Reconocimiento-NoComercial-Compartirigual 4.0 International License. Creado a partir de la obra en http://www.revistas. ucr.ac.cr/index.php/matematica

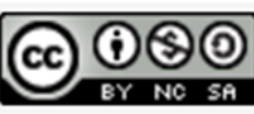

*Universidad Autónoma Metropolitana-Iztapalapa, Departamento de Economía, Ciudad de México, México. E-Mail: jcdesprof@gmail.com

†Universidad Autónoma Metropolitana-Iztapalapa, Departamento de Ingeniería Eléctrica, Ciudad de México, México. E-Mail: gamma@xanum.uam.mx

${ }^{\ddagger}$ Universidad Autónoma Metropolitana-Iztapalapa, Departamento de Matemáticas, Ciudad de México, México. E-Mail: eomagna@gmail.com

${ }^{\S}$ Misma dirección que/Same address as: M. Gutiérrez-Andrade. E-Mail: cobos@xanum.uam.mx
\end{abstract}




\begin{abstract}
Resumen
El objetivo de este trabajo es evaluar la eficacia de una política ambiental que promueva la sustitución de energías fósiles por renovables, con la finalidad de reducir gradualmente las emisiones de dióxido de carbono a lo largo de un periodo. Se utiliza un modelo dinámico de equilibrio general computable para la economía mexicana y se simulan escenarios con dos elasticidades de sustitución, cuando se gravan de manera conjunta a dos de los bienes energéticos que usan combustibles fósiles, midiendo sus impactos sobre la producción agregada y el bienestar social. El análisis de los resultados muestra que la eficacia de la política depende de la elasticidad de sustitución. En el escenario rígido es parcialmente efectiva, ya que se reducen las demandas de todas las energías, fósiles y renovables. El escenario flexible resulta ser eficaz, al favorecer la sustitución entre las energías. La reducción es mayor en el escenario rígido que en el flexible. En cada escenario al incrementar el impuesto disminuyen el PIB y el bienestar social respecto al equilibrio de referencia.
\end{abstract}

Palabras clave: política ambiental; desarrollo sustentable; equilibrio general computable; energía; modelos dinámicos.

\begin{abstract}
The objective of this work is to evaluate the effectiveness of an environmental policy that promotes the substitution of fossil fuels for renewable alternatives, to reduce carbon dioxide emissions. A dynamic computable general equilibrium model is used for the Mexican economy simulating economic scenarios with two elasticities of substitution, when two of the energy goods that use fossil fuels are taxed together. The impacts on aggregate production and social well-being are quantified or measured. The analysis shows that the effectiveness of the policy depends on the elasticity of substitution. The rigid scenario is partially effective, since the demands of all the energies, fossils and renewable are reduced. The flexible scenario turns out to be effective, favoring the substitution between energies. The demand of all energies is further reduced in the rigid scenario than in the flexible. In each scenario, increasing the tax reduces GDP and social welfare in relation to the reference equilibrium.
\end{abstract}

Keywords: environmental policy; sustainable development; computable general equilibrium; energy; dynamic models.

Mathematics Subject Classification: 90C33, 90C46, 91B50. 


\section{Introducción}

La contaminación del aire provocada por el uso de los combustibles fósiles tiene un impacto negativo en el calentamiento global del planeta y afecta a la salud de la población. Se considera un gran riesgo para la salud humana, incluso la tasa de mortalidad que está relacionada con la mala calidad del aire es una de las mayores del mundo, sobre todo en centros urbanos debido principalmente al uso de vehículos automotores de combustión interna y a las actividades industriales intensivas en el uso de energía [17, 30].

El cambio climático es consecuencia de una externalidad negativa global originada por las emisiones de gases de efecto invernadero (GEI) a la atmósfera. Se estima que la temperatura media del mundo podría aumentar hasta tres grados centígrados en este siglo. Las emisiones de gases de efecto invernadero (GEI), causadas por las actividades humanas, hacen que esta amenaza aumente. Entre sus consecuencias se incluyen los impactos negativos en la economía y cambios en los patrones climáticos, el aumento del nivel del mar y los fenómenos meteorológicos extremos.

En la búsqueda de soluciones a esta problemática, casi 150 países adoptaron el Acuerdo de París sobre cambio climático en diciembre de 2015, comprometiéndose a presentar planes voluntarios de reducción de emisiones para impedir que la temperatura suba más de dos grados a finales de siglo. La cooperación internacional busca que la actividad económica de los países sea más sostenible y respetuosa con el medio ambiente.

Uno de los principales retos consiste en evitar o mitigar los impactos del calentamiento global y que los países avancen hacia una economía baja en dióxido de carbono $\left(\mathrm{CO}_{2}\right)$, a través de políticas que favorezcan el uso de la energía renovable y otras soluciones para reducir las emisiones, sin impedir el crecimiento económico, sobre todo de los países menos desarrollados.

En la actualidad, América Latina y el Caribe producen el 5\% de las emisiones mundiales de GEI. No obstante, su contribución a las cifras mundiales está aumentando, a consecuencia sobre todo de las demandas impuestas de energéticos por el sector industrial y el de transporte. Las emisiones de $\mathrm{CO}_{2}$ procedentes de la quema de combustibles fósiles y la fabricación de cemento en la región, aumentaron $14.2 \%$ durante el periodo de 2006 a 2011, aunque su proporción en relación con el producto interno bruto (PIB) ha disminuido, por lo que reducir las emisiones de GEI se considera un reto importante para la región y los contaminantes como el carbono negro han pasado a ser una prioridad en la actualidad [34]. 
En México, el dióxido de carbono $\left(\mathrm{CO}_{2}\right)$ representó en el 2013, el $75.1 \%$ del total de los GEI, seguido por el metano $\left(\mathrm{CH}_{4}\right)$ con un $19 \%$, el óxido de nitrógeno $\left(\mathrm{N}_{2} \mathrm{O}\right)$ con $4.5 \%$ y los clorofluorocarbonados con $1.4 \%$; de 1990 a 2012, las emisiones de $\mathrm{CO}_{2}$ se incrementaron $33 \%$, en cambio las de $\mathrm{CH}_{4}$ lo hicieron $32 \%$, el $\mathrm{N}_{2} \mathrm{O}$ el $5 \%$ y los clorofluorocarbonados $92 \%$. Se estima que alrededor del $85 \%$ de las emisiones de $\mathrm{CO}_{2}$ provinieron del uso de los combustibles fósiles en el sector de energía [17].

La demanda de energía en México ha estado históricamente relacionada con el crecimiento económico y se estima que el PIB permanecerá como un gran contribuyente a la demanda de energía. La vulnerabilidad de nuestro país ante los impactos del cambio climático, se manifiesta especialmente en la población de bajos ingresos, pero se considera que más de dos terceras partes de su población ha sido impactada por desastres naturales como sequías e inundaciones [18].

En México, la Ley General de Cambio Climático establece metas para transitar hacia una economía baja en carbono, con el fin de garantizar el derecho a un medio ambiente sano, desarrollo sustentable, así como a la preservación y restauración del equilibrio ecológico.

En esta misma ley se acuerda alcanzar la meta de reducir $50 \%$ las emisiones de GEI para el año 2050, en relación con las emitidas en el año 2000; también se establece el objetivo de lograr 35\% de la generación de energía eléctrica con base en energías limpias para el año 2024 [30].

Entre los incentivos fiscales más sobresalientes que aplican los gobiernos para la promoción de las energías limpias y el uso eficiente de la energía, está el impuesto al carbono, el cual incrementa su precio y envía una señal para reducir su uso en la economía y de sus emisiones, por medio de acciones que incluyen la eficiencia económica y energética. En algunos casos, se trata de un impuesto al consumo de energía, lo cual incentiva directamente la eficiencia [30, 17].

Las políticas de desarrollo requieren de la realización de análisis de prospectiva sectorial, así como elaboración de estrategias, planes y programas relacionados con el desarrollo sustentable y el medio ambiente, incluyendo la estimación de los costos futuros asociados al cambio climático, así como los beneficios derivados de las acciones para enfrentarlo. El análisis requiere también que se evalúen los costos y los beneficios de este tipo de políticas y se muestre cuáles son los sectores que podrían realizar el cambio hacia tecnologías no contaminantes.

En el caso de México, a pesar de que la producción de las energías renovables se ha incrementado durante los últimos años, los combustibles fósiles -petróleo, gas y carbón principalmente- representaron el $91.1 \%$ de la oferta nacional de energía, en el año 2015 [30]. 
Este trabajo tiene como objetivo, evaluar la eficacia de una política ambiental que promueva la sustitución de energías tradicionales o de origen fósil, por energías renovables, con la finalidad de reducir las emisiones de dióxido de carbono $\left(\mathrm{CO}_{2}\right)$ a la atmósfera, así como evaluar sus costos a través de las repercusiones o impactos en la producción agregada y el bienestar social, en el marco de un modelo dinámico de equilibrio general computable.

En este trabajo se utiliza un modelo dinámico tipo Ramsey en el que se gravan los insumos energéticos, para evaluar el impacto de esta política en el medio ambiente, las variables macroeconómicas y en el bienestar de la familia representativa. Las simulaciones se llevan a cabo para dos posibles valores en la elasticidad de sustitución; para cada uno de estos se mide la sustitución de las energías fósiles, con altas emisiones de dióxido de carbono, por energías renovables.

Se considera un escenario base en el que la senda del crecimiento ocurre de manera inercial, en este se toma la estructura económica inicial, distorsionada con los impuestos, subsidios y transferencias, y se compara con otros dos escenarios correspondientes a dos elasticidades de sustitución (rígida y elástica), al modificarse la estructura productiva y sustituir los insumos energéticos contaminantes por los no contaminantes.

Se sabe que toda política fiscal que genere beneficios ambientales ocasiona costos a la sociedad, básicamente puede limitar la actividad económica en general e impactar negativamente al bienestar social, aunque esto último puede modificarse bajo ciertas condiciones y obtener un doble dividendo en mejoras ambientales y bienestar [8, 13].

En particular, la política fiscal considera el incremento de impuestos a la demanda de energías intermedias de origen fósil en los diferentes sectores energéticos, extracción de petróleo y gas (EPG), y en la fabricación de productos derivados del petróleo y del carbón (FPPC). Posteriormente, se simulan los escenarios con distintas elasticidades de sustitución a lo largo del tiempo.

Desde el punto de vista ambiental, la política incentiva la sustitución de energías tradicionales o no renovables por un bien compuesto denominado energías renovables (REN), en el cual, se considera tanto la energía eólica como la solar y la hidráulica, en particular, este bien renovable sólo es utilizado para la producción del bien energético Generación de Energía Eléctrica (GEE). La simulación de la política de acuerdo con el modelo utilizado, permite analizar la respuesta de la economía para verificar si la política ambiental resulta eficaz o no, para disminuir las emisiones de carbón a la atmósfera. 
Las emisiones de $\mathrm{CO}_{2}$ a la atmósfera, se reducen debido a las variaciones en los precios relativos de los insumos, que a su vez originan cambios en la cantidad demandada del bien energético agregado (E), al sustituir el uso de los bienes energéticos por otros bienes intermedios y factores de la producción. En particular, las empresas sustituyen bienes energéticos fósiles que son intensivos en $\mathrm{CO}_{2}$ por otros menos intensivos.

En la siguiente parte se reseña la literatura relevante. El marco teórico se describe en la Sección 2, en la Sección 3 se muestran los resultados obtenidos y el análisis correspondiente; en la parte final se presentan las conclusiones y algunas recomendaciones derivadas del análisis de los resultados.

\section{Estado del arte}

Los modelos de equilibrio general computable permiten realizar simulaciones de respuestas de las variables endógenas ante choques exógenos, principalmente de política económica. Se utilizan ampliamente en el área de la energía, la economía y el medioambiente, así como en la planeación y el diseño de políticas energéticas, sustitución de tecnologías contaminantes, explotación de tierra asignada al cultivo para obtener bioenergéticos, etc [2].

Existe una gran variedad de este tipo de modelos, algunos se basan en el diseño de políticas fiscales que penalizan el desperdicio y la contaminación generada por el uso de energéticos de origen fósil, en otros se verifica el uso de los energéticos renovables y bioenergéticos, ver por ejemplo [5, 15]. Destaca un excelente estudio de [2] sobre la planeación de los recursos energéticos, en el cual, mediante el uso combinado de las metodologías "bottom-up" y "top-down”, realiza un análisis sobre la sustitución y la conversión de tecnologías más allá de las formas funcionales que puedan asumir.

Una parte sustancial de la discusión sobre las políticas para reducir las emisiones de dióxido de carbono y otros gases de efecto invernadero, se centra en las políticas que reduzcan al mínimo los costos, las cuales son deseables porque son económicamente más eficientes; entre las distintas políticas de costo mínimo, destaca la de impuestos sobre el carbono [14]. Resulta importante medir los efectos positivos de la política con respecto a la sustitución de las energías de origen fósil por las renovables, así como sus costos, es decir, los impactos negativos sobre la producción, el empleo y el bienestar social.

Jensen y Rasmussen [12] muestran los impactos negativos, principalmente en los niveles de empleo y del bienestar social, que derivan al aplicar una política de asignación de permisos negociables otorgados para reducir las emisiones de carbono. Los resultados indican que el costo en el bienestar es menor, en cambio 
la reducción en el empleo es significativa, sobre todo en los sectores intensivos en energía. Además, se manifiesta que la distribución de los permisos, en función de las cuotas de mercado, disminuye los ajustes de la economía sectorial, aunque con un alto costo en el bienestar.

En un trabajo similar, González-Eguino [7] utiliza un modelo de equilibrio general dinámico computable, tipo Ramsey, para simular las políticas en el largo plazo, dirigidas a la mitigación de gases de efecto invernadero en España. Se estudia la evolución de las principales variables macroeconómicas y sectoriales, así como la variación de los insumos energéticos y del precio de los permisos de emisión. Los resultados muestran que los costos de cumplir con los objetivos de emisiones signados en el Protocolo de Kyoto, podrían limitarse si se induce un cambio hacia una economía menos intensiva en carbono, a través de mejoras en la eficiencia energética y fundamentalmente, mediante la innovación tecnológica.

Los modelos de equilibrio general computable (MEGC), han sido utilizados con frecuencia para analizar los impactos económicos y medioambientales del establecimiento de un impuesto al carbón. En [33] utilizan un modelo estático, en el caso de Tailandia, y encuentran que el bienestar y el producto agregado disminuyen, pero sorprendentemente casi no hay reducción de las emisiones de carbono.

En [31], se utiliza un MEGC para estudiar las perspectivas en Noruega si las emisiones de $\mathrm{CO}_{2}$ fueran controladas. Los resultados muestran que las emisiones de $\mathrm{CO}_{2}$ podrían estabilizarse sin reducir drásticamente el crecimiento económico, aunque los efectos sobre la asignación sectorial son mucho mayores, también se produce un aumento en el bienestar económico.

Yuan y Lu [35] analizan el efecto del impuesto sobre las emisiones de carbono en la economía China mediante un MEGC dinámico. Los resultados de la simulación muestran que un impuesto tiene impactos negativos en el crecimiento económico únicamente en el corto plazo, ya que a largo plazo se logran reducir las emisiones de carbono mientras se estimula el desarrollo económico.

También, en [10] analizan mediante un MEGC las posibles consecuencias que resultan sobre la economía china y la variación de las emisiones de carbono al aplicar un impuesto al carbono. Los resultados empíricos indican que un impuesto moderado al carbono permite reducir significativamente las emisiones de carbono y el consumo de energía de combustibles fósiles, mientras el ritmo de crecimiento económico se reduce un poco. Sin embargo, un impuesto grande al carbono tendría un impacto significativamente negativo en la economía y el bienestar social. La conclusión principal es que un impuesto al carbono promueve el uso de energías limpias, lo cual es un medio eficaz para reducir las 
emisiones de carbono y contribuir a la mitigación de los efectos nocivos del cambio climático.

En [32] se encuentran resultados similares para la economía China, en este se analiza la reforma fiscal implementada por el gobierno chino en diciembre de 2014 para desarrollar una economía baja en carbono. Mediante un MEGC dinámico, se analiza la política de impuestos al carbón y sus impactos sobre la economía y el medio ambiente. Los resultados muestran que el PIB se afecta, así como la producción en la mayoría de los sectores, aunque estos efectos disminuyan con el paso del tiempo; adicionalmente, se presenta una fuerte reducción en el carbono, al mismo tiempo que se incrementa el uso de las energías limpias.

En el caso de México, Romero [24] muestra que las emisiones totales de carbón disminuyen $13 \%$ cuando se grava con $20 \%$ el uso del carbón, mientras que los impactos sobre el índice de precios y el PIB son mínimos.

En [3] se utiliza un modelo de MEGC para evaluar los efectos de un impuesto al carbono para el caso mexicano y concluyen que esta política disminuye la intensidad de las emisiones de carbono sin pérdidas significativas en el bienestar social.

Un modelo MEGC dinámico, en [16] es utilizado para estimar el valor del impuesto al carbón que se requiere para cumplir con los objetivos de la reducción de emisiones, comprometidos en la legislación mexicana del cambio climático. En este trabajo se simulan los efectos, de mediano y largo plazo, que produce la aplicación de esta política energética sobre el medioambiente y la economía mexicana. Los resultados muestran que, si no hay compensación del impuesto, se reducirían las emisiones de $\mathrm{CO}_{2}$ en más del $75 \%$ para el año 2050 con respecto al escenario base, pero a un alto costo, ya que se provocaría una recesión económica. Si en cambio se redistribuyen los impuestos, principalmente se reduce el impuesto sobre la renta a las familias, generándose un doble dividendo, el efecto sobre el PIB es positivo y se logra reducir las emisiones de $\mathrm{CO}_{2}$.

Se concluye que es posible aplicar un impuesto al carbón que cause un efecto positivo para el medioambiente, al reducir las emisiones de $\mathrm{CO}_{2}$, aceptable desde la perspectiva económica y política. Este resultado depende de la capacidad de la economía para adaptarse al cambio en los precios relativos de los energéticos, es decir, del nivel que tenga la elasticidad de sustitución asumida en la simulación.

También, en [4] evalúan mediante un MEGC estático el efecto sobre la economía mexicana al aplicar un impuesto a la demanda de combustibles fósiles, con el fin de disminuir la emisión de dióxido de carbono $\left(\mathrm{CO}_{2}\right)$ a la atmósfera. 
Las simulaciones muestran que la efectividad de la política aumenta en la medida en que hay una mayor elasticidad de sustitución técnica entre las energías, es decir, que la sustitución de las energías contaminantes por el uso de energías renovables, depende de la elasticidad de sustitución que exista en la economía.

\section{Marco teórico}

Los MEGC están fundamentados, por un lado en el marco de la microeconomía y macroeconomía, y por otro lado, en el contexto formal de la optimización y de la teoría del cálculo variacional. Esto se debe a que algunos de los MEGC incluyen modelos de optimización y otros no. Si los MEGC incorporan modelos de optimización, entonces las condiciones de primer orden, holgura complementaria y las condiciones de equilibrio económico de cero beneficio, vaciado de mercados e ingresos balanceados; definen un conjunto de igualdades y desigualdades no lineales que pueden ser resueltas por algoritmos y métodos propios de la investigación de operaciones, estudiados y desarrollados específicamente para problemas de optimización.

La matriz de contabilidad social (SAM), es un sistema de información estructurado en el que se reflejan las actividades económicas, una parte refleja la interacción de los sectores productivos, en la matriz de insumo producto; se estratifica a los diferentes consumidores de acuerdo a sus ingresos y preferencias de consumo, se contabilizan los ingresos del gobierno como recaudador de impuestos y sus egresos como proveedor de bienes y servicios, también se contabilizan los ingresos y egresos de un país ocasionados por tratados de comercio nacional e internacional. En general, una matriz SAM se interpreta por renglón como los ingresos del sector o agente correspondiente a la fila, de manera análoga, las columnas representan sus egresos. En el Anexo (Secciï ¿ $\left.{ }^{1 / 2 n} 5\right)$ se muestra la matriz utilizada.

El MEGC de este trabajo utiliza una matriz SAM (ver Anexo) que se fundamenta en la construida por [1], quienes emplean la Matriz de Insumo-Producto, (2004) del INEGI y la Encuesta nacional de ingresos y gastos de los hogares. A esta matriz se le agregó información contenida en documentos oficiales de la Secretaría de Energía [29], correspondiente con la producción de las energías renovables como un bien intermedio.

El modelo propuesto representa una economía abierta en competencia perfecta en todos los mercados, hay ocho agentes: seis industrias, la familia y el gobierno. Existen cinco bienes de consumo final, seis bienes intermedios, y como factores de producción, el trabajo y el capital. 
Los sectores productivos se dividen en sectores no energéticos SPR (bien primario) e ISE (bien secundario) y los sectores energéticos que producen energías fósiles son: la extracción de petróleo y gas (EPG), y la fabricación de productos derivados del petróleo y del carbón (FPPC). Existen otros dos sectores energéticos, uno de ellos es el sector de generación de energía eléctrica (GEE), y por último está el sector de energías renovables (REN) que abastece de manera única al sector GEE de energías renovables como insumos.

Para hacer una proyección a mediano plazo es más eficiente utilizar los modelos dinámicos, por lo que en este trabajo se recurre concretamente a un modelo tipo Ramsey, que corresponde a un modelo clásico de la macroeconomía, y frecuentemente se usa en los MEGC dinámicos (consultar [28]). Estos modelos permiten determinar las variables que definen el producto interno bruto PIB, sin olvidar que permite optimizar la función de utilidad del consumidor representativo de manera intertemporal. Existen diferentes aproximaciones a los modelos dinámicos, ver [11, 22, 6, 9].

Las hipótesis generales del modelo suponen que la familia representativa maximiza la utilidad obtenida en cada periodo de tiempo $t$. Los agentes son un consumidor que tiene una vida infinita, seis productores y el gobierno. En el contexto de la optimización, los modelos tipo Ramsey están representados como modelos de Programación no Lineal, y por su uso en los modelos de equilibrio son analizados en el marco de los modelos complementarios mixtos, que resultan útiles, ya que se les pueden incorporar políticas de ahorro e inversión, condiciones que requieren los modelos de equilibrio dinámico. En cada periodo $t$, cada industria produce a través de un proceso anidado. El modelo utilizado en este trabajo es determinista, el tiempo se considera una variable discreta y se supone un horizonte de planeación infinito, requiere condiciones de tipo macroeconómico que incluyen: condiciones iniciales sobre el capital, condiciones de crecimiento del trabajo y condiciones de cerradura que conduzcan a la solución a un estado estacionario a partir del intervalo de tiempo $t=T$, ya que el modelo utiliza un horizonte de planeación infinito. Para cada intervalo de tiempo se definen y describen las condiciones de equilibrio a ser satisfechas.

Los modelos correspondientes para el productor y el consumidor se tienen que resolver simultáneamente en términos de un sistema de ecuaciones complementarias, de manera conjunta con las condiciones de equilibrio para cada periodo $t$.

El modelo correspondiente al productor, se resuelve para cada periodo de tiempo; sin embargo, el modelo del consumidor se presenta para describir cómo está involucrado su dual y cómo se puede representar a través de dos problemas, uno de ellos con horizonte finito. 
Se utiliza un modelo anidado para representar el proceso productivo de cada uno de los sectores y se modela con una función Cobb-Douglas. Existe un bien intermedio llamado energía agregada, que se obtiene a partir de los bienes energéticos y se modela mediante una función de elasticidad constante (CES), ver Figura 1. Las variables referidas en la Figura 1 se declaran en las subsecciones $3.1 .1,3.1 .2,3.1 .3$ y 3.2 .

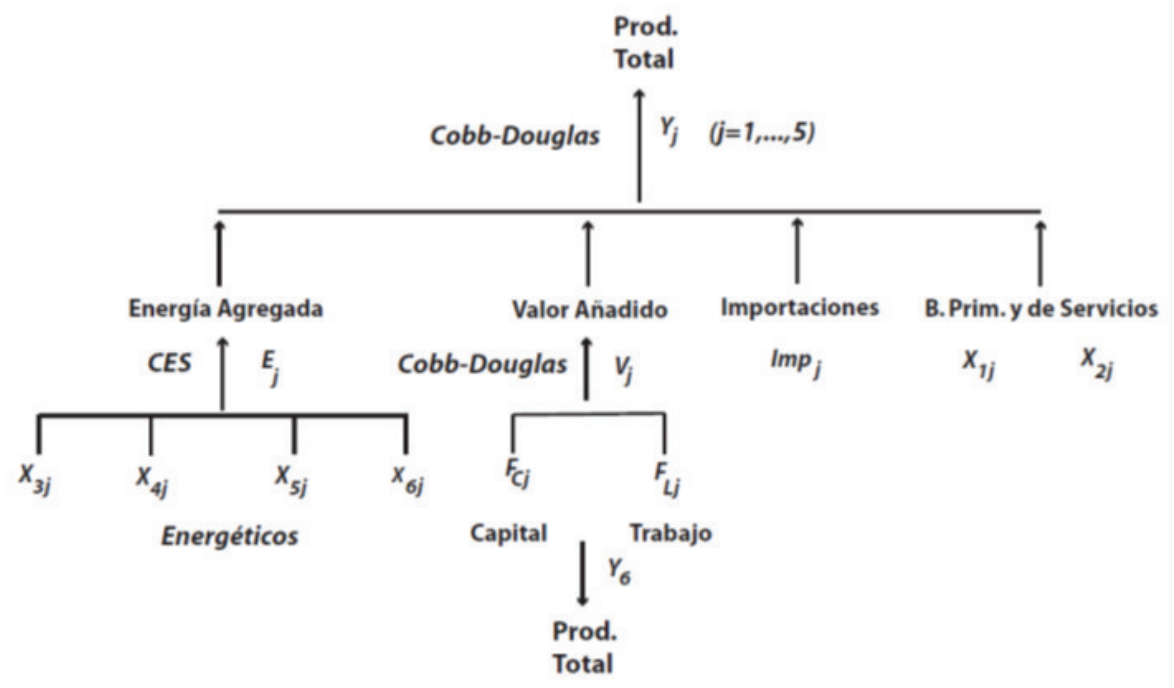

Figura 1: Comportamiento anidado de los productores.

\subsection{Modelo anidado}

La anidación consiste en representar el proceso de producción para cada empresa como un árbol (Figura 1), los vértices del nivel bajo se representan a los factores de producción y a los bienes energéticos. Los factores de producción se utilizan para elaborar el valor añadido por medio de una función Cobb-Douglas, los bienes energéticos sirven para producir la energía agregada a través de una función de elasticidad constante CES, que supone cierta elasticidad de sustitución. En ambos casos se persigue producir a costo mínimo. En el siguiente nivel, se encuentran como nodos intermedios los bienes primarios, los bienes de la industria y servicios, las importaciones, el valor añadido ligado a los factores y la energía agregada conectada a los energéticos. Todos estos vértices representan los bienes requeridos para llevar a cabo la producción total de cada industria, la que aparece como un nodo raíz. Se supone que la producción total de cada bien se rige utilizando una función Cobb-Douglas. Algunas referencias relacionadas con el uso de este proceso para el sector energético de USA se encuentran en [20, 23]. 


\subsubsection{Primera etapa: producción del valor añadido}

La familia representativa ofrece el trabajo (índice $L$ ) y capital (índice $C$ ) para que las empresas utilicen estos factores a través de una función Cobb-Douglas en la producción del valor añadido, que debe alcanzar cierto nivel de producción. El modelo para cada periodo de tiempo es:

$$
\min \sum_{k=L}^{C} w_{k} F_{k j},
$$

sujeto a $V_{j}=B_{j} \Pi_{k=L}^{C} F_{k j}^{\beta_{k j}}$, donde $\sum_{k=L}^{C} \beta_{k j}=1$ para $\beta_{k j} \in[0,1]$.

La función objetivo representa el costo de los factores, los cuales combinados determinan el nivel de producción del valor añadido $V_{j}$ que queda establecido en la restricción.

Notación:

$V_{j}$ denota el valor añadido a cada uno de los bienes producidos, para $j=1, \ldots, 6$.

$F_{k j}$ es la demanda del factor de producción $k$ para producir el valor añadido $V_{j}$, para $k=L, C$ y para $j=1, \ldots, 6$.

$B_{j}$ es el coeficiente de escala en la función de producción del valor añadido.

$\beta_{k j}$ es el coeficiente de proporcionalidad del factor $F_{k j}$, para $k=L, C$ en la función de producción del valor añadido $V_{j}$, para $j=1, \ldots, 6$.

$w_{k}$ es el precio del factor $F_{k j}$, para $k=L, C$.

\subsubsection{Segunda etapa: producción de la energía agregada}

Para precisar el modelo que sirve para determinar la producción de energía agregada $E_{j}$, para $j=1, \ldots, 5$ para cada empresa, se supone que $E_{j}$ está compuesta solamente por los bienes energéticos y dado que el objetivo es sustituir a las energías fósiles por renovables, se supone que la demanda $X_{i j}$ de estos bienes intermedios se representa por medio de una función CES.

El subíndice $i$ solamente debe considerar los bienes energéticos representados en los renglones $i=3, \ldots, 6$ de la SAM, y debido a que todas las empresas excepto la productora de energías renovables utilizan la energía agregada, el subíndice $j$ satisface que $j=1, \ldots, 5$. Finalmente, la elasticidad de sustitución entre los bienes intermedios $X_{i j}$ está definida por el parámetro $\sigma_{j}$. Si el valor de 
este parámetro es menor que uno, se dice que hay rigidez en la sustitución y si es mayor que uno, se dice que es flexible, cabe aclarar que el parámetro de elasticidad no aparece explícitamente en el modelo. Entonces, el modelo que permite determinar el nivel de producción de la energía agregada, para cada periodo de tiempo y que define el nivel de sustitución de los bienes energéticos intermedios, está dado por:

$$
\min \sum_{i=3}^{6} p_{i} X_{i j}
$$

sujeto a:

$$
E_{j}=\psi_{j}\left(\sum_{i=3}^{6} \gamma_{i j} X_{i j}^{\rho_{j}}\right)^{\frac{1}{\rho_{j}}}
$$

en donde $\sum_{i=3}^{6} \gamma_{i j}=1$ para $\gamma_{i j} \in[0,1]$.

Notación:

$p_{i}$ denota el precio de la energía intermedia $X_{i j}$, para $i=3, \ldots, 6$ que requiere la producción de la energía agregada del sector $j=1, \ldots, 5$.

$E_{j}$ es el nivel de la energía agregada que demanda el sector $j=1, \ldots, 5$.

$\gamma_{i j}$ denota el coeficiente de proporcionalidad del bien intermedio $X_{i j}$ en la función de producción de la energía agregada $E_{j}$, para $i=3, \ldots, 6$.

$\rho_{j}=\frac{\sigma_{j}-1}{\sigma_{j}}$ es el parámetro definido por la elasticidad de sustitución $\sigma_{j}$.

$\psi_{j}$ es el coeficiente de escala en la función de producción de la energía agregada.

\subsubsection{Tercera etapa: producción óptima para cada sector}

El modelo a resolver para cada empresa y cada periodo de tiempo, tiene como objetivo minimizar los costos de producción sujeto a que la producción total alcanza un nivel de producción $Y_{j}$ definido por una función Cobb-Douglas, que se distingue porque el valor de la elasticidad es justamente uno.

$$
\min C_{j}=v_{j} V_{j}+\sum_{i=1}^{2} p_{i} X_{i j}+e_{j} E_{j}+p_{j} I m p_{j}
$$

sujeto a:

$$
Y_{j}=D_{j}\left(\Pi_{k=1}^{2} X_{k j}^{\iota_{k j}}\right) V_{j}^{\iota_{v j}} E_{j}^{\iota_{e j}} \operatorname{Im} p_{j}^{\iota_{i j}}
$$


en donde $\sum_{k=1}^{2} \iota_{k j}+\iota_{v j}+\iota_{e j}+\iota_{i j}=1$, para los valores de $\iota_{k j}, \iota_{v j}, \iota_{e j}$, y $\iota_{i j} \in[0,1]$ y formalmente se resuelve para $j=1, \ldots, 5$.

Notación:

$Y_{j}$ es la cantidad total de la producción de la industria $j=1, \ldots, 5$.

$C_{j}$ representa los costos totales de producción del bien final $Y_{j}$.

$D_{j}$ denota el coeficiente de escala en la función de producción.

$\iota_{k j}$ representa el coeficiente de proporcionalidad del bien intermedio $X_{k j}$ en la función de producción del bien final $Y_{j}$, para $k=1,2$.

$\iota_{v j}, \iota_{i j} \mathrm{y} \iota_{e j}$ denotan respectivamente el coeficiente de proporcionalidad del valor añadido $V_{j}$, el coeficiente de proporcionalidad del bien intermedio de importación $I m p_{j}$ y el coeficiente de proporcionalidad de la energía agregada $E_{j}$, para producir una unidad de la producción total $Y_{j}$.

\subsection{Modelo para el consumidor}

La familia representativa o consumidor tiene como objetivo maximizar una función de utilidad tipo Cobb-Douglas (consultar [19, 20, 27]) en un horizonte de tiempo infinito. En cada periodo de tiempo, la familia obtiene sus ingresos a partir de la renta del trabajo y de su capital inicial. Se supone que el trabajo crece a una tasa constante que depende de la tasa de crecimiento de la población y el valor inicial del capital está determinado por la dotación inicial de la familia, y se denota por $F_{C}^{0}=F_{C}$. Existen transferencias a la familia, realizadas por el gobierno $T_{g}^{t}$ y por el sector externo $T_{e}^{t}$, respectivamente; en cada periodo $t$ gastan sus ingresos en los bienes de consumo final $X_{i}^{t}$, pagan impuestos al gobierno y ahorran.

Finalmente, el gobierno que actúa como un consumidor pasivo, obtiene sus ingresos por la recaudación de impuestos que posteriormente utiliza en el consumo de bienes finales, pago de factores de producción, transferencias a las familias e importaciones. 
El modelo para los consumidores, consiste en maximizar la función de utilidad:

$$
U\left(X_{1}, \ldots, X_{5}\right)=\sum_{t=0}^{\infty}\left(\frac{1}{1+\rho}\right)^{t} \prod_{i=1, i \neq 4}^{5}\left(X_{i}^{t}\right)^{\alpha_{i}}
$$

sujeto a:

$$
\sum_{t=0}^{\infty}(1+\tau)\left(\sum_{i=1}^{5} p_{i}^{t} X_{i}^{t}+S_{p}^{t}\right)=\omega_{C}^{0} F_{C}^{0}+\sum_{t=0}^{\infty}\left(\omega_{L}^{t} F_{L}^{t}+T_{g}^{t}+T_{e}^{t}\right),
$$

donde $\sum_{i=1}^{5} \alpha_{i}=1$ y $\alpha_{i} \in[0,1]$.

Notación:

$X_{i}^{t}$ es la demanda del bien final de la familia representativa, para $i=1,2,3,5$ donde $i=1$ representa el bien SPR, $i=2$ a ISE, $i=3$ a FPPC e $i=5$ a GEE en el periodo $t$.

$p_{i}^{t}$ son los precios de los bienes de consumo final en el periodo $t$.

$\alpha_{i}$ representan los coeficientes de participación, del bien final $i=1, \ldots, 5$ en la función de utilidad de la familia.

$S_{p}^{t}$ denota el ahorro de la familia en el periodo $t$.

$F_{L}^{t}$ es la dotación del trabajo del consumidor en el periodo $t$.

$\omega_{L}^{t}$ denota el costo del trabajo en el periodo $t$.

$\tau$ es la tasa que grava el consumo total de la familia.

$T_{g}^{t}$ son las transferencias del gobierno a la familia en el periodo $t$.

$T_{e}^{t}$ denotan las transferencias del sector externo a la familia en el periodo $t$.

\subsubsection{Condiciones intertemporales e iniciales}

Las condiciones macroeconómicas son intertemporales y determinan el crecimiento de la economía, estas condiciones están relacionadas con el consumo, producción e inversión agregadas, [19]. En una economía abierta con sector gobierno, se cumple la siguiente identidad:

$$
X^{t}=Y^{t}-I^{t}-X_{g}^{t}-\left(E x p^{t}-I m p^{t}\right),
$$


donde: $X^{t}$ es el consumo agregado, $Y^{t}$ es la producción agregada, $I^{t}$ la inversión, $X_{g}^{t}$ es el gasto público y $E x p^{t}-I m p^{t}$ es la balanza comercial.

En el caso de los modelos dinámicos, el crecimiento del capital $F_{C}^{t+1}$ para cada periodo $t+1$ está determinado por el valor del capital $(1-\delta) F_{C}^{t}$ en el periodo $t$ más las inversiones correspondientes $I^{t}$, en donde $\delta$ denota la depreciación del capital. Esta condición se integra al modelo mediante la restricción $F_{C}^{t+1}=(1-\delta) F_{C}^{t}+I^{t}$ y debe ser satisfecha en cada periodo $t+1$.

Para el caso particular en que $t=0$, se define el valor inicial del capital como la dotación de la familia representativa, $F_{C}^{0}=F_{C}$, de este factor, la inversión total inicial $I^{0}$ se obtiene como la suma de las inversiones iniciales de cada sector $I n v_{j}^{0}$ más la transferencia del ahorro al sector externo $T A_{e}^{0}$ :

$$
I^{0}=\sum_{i=1}^{5} \operatorname{Inv} v_{j}^{0}+T A_{e}^{0} .
$$

Para determinar el factor trabajo en cada periodo $t$, se requiere conocer el trabajo inicial $F_{L}^{0}$ que corresponde la dotación de la familia $F_{L}$, por lo que $F_{L}^{0}=$ $F_{L}$. Respecto al crecimiento del trabajo en la literatura, existen diferentes formas de crecimiento, aquí se supone que crece a una tasa constante $g$. En general, el crecimiento del trabajo para cada periodo de tiempo está determinado por $F_{L}^{t}=(1+g)^{t} F_{L}^{0}$ o de manera equivalente $F_{L}^{t}=(1+g) F_{L}^{t-1}$.

\section{Resultados}

Entre las herramientas computacionales que se han desarrollado para resolver tanto los problemas estáticos como dinámicos, Alex Meeraus inicia el desarrollo del modelador General Algebraic Modelin System (GAMS) y funda la Corporación de Desarrollo GAMS, este lenguaje de modelado algebraico fue evolucionando para resolver problemas de programación matemática y de condiciones complementarias para el caso estático. En particular, para los MEGC dinámicos, Rutherford utiliza las condiciones complementarias para un modelo tipo Ramsey estable (ver [26, 25, 27]) en el sentido de que varias cantidades (capital, producción, consumos, etc.) crecen a razón constante y posteriormente su alumno Paltsev [19] expone un análisis y comparación de los modelos estáticos y dinámicos, y el uso del paquete GAMS para resolver ambos casos; para el caso dinámico, los modelos son del tipo Ramsey y con un horizonte de planeación infinito. En particular, [27] presenta cómo los problemas complementarios mixtos pueden ser resueltos con el simulador GAMS.

Los resultados se analizan e interpretan cuando se impone un impuesto simultáneo a los dos bienes energéticos de origen fósil, EPG y FPPC. Los valores 
de los impuestos son 5\%, $10 \%$ y 15\%; el horizonte de planeación es de 25 años. Las tablas reportan los resultados para los años 15 y 25 . Los valores de la elasticidad son $\sigma=0.5$ y $\sigma=1.8$, que reflejan un escenario rígido y uno elástico; estos valores fueron utilizados por [20, 23, 21, 4] para diferentes estudios. En la Tabla 1 se muestran los valores de la elasticidad de sustitución utilizados en estos estudios.

Tabla 1: Elasticidades de sustitución técnica.

\begin{tabular}{c|ccc}
\hline \hline $\begin{array}{c}\text { Sectores } \\
\text { Energéticos }\end{array}$ & $\begin{array}{c}\text { Elasticidad de } \\
\text { sustitución }\end{array}$ & Fuente & País \\
\hline GEE, EPG, FPPC y REN & 0.5 & {$[20,23]$} & Estados Unidos \\
GEE, EPG, FPPC y REN & 1.8 & {$[21]$} & Mundial \\
GEE, EPG, FPPC y REN & 0.5 y 1.8 & {$[4]$} & México \\
\hline \hline
\end{tabular}

Se presenta primero una comparación del comportamiento dinámico de las variables macroeconómicas, así como el nivel de bienestar bajo dos escenarios y posteriormente se analiza la evolución temporal de las demandas de insumos energéticos.

\subsection{Efectos de los impuestos sobre las variables macroeconómicas para $\sigma=0.5$}

La tabla 2 resume los resultados de la simulación de una política de impuestos a las energías contaminantes, durante un periodo temporal de 25 años en el primero de los escenarios: una economía con rigidez en la sustitución técnica $(\sigma=0.5)$ de los insumos energéticos. En este cuadro, se compara la tasa de crecimiento de las principales variables macroeconómicas respecto al escenario base o inercial, en el cual se representa una economía donde está ausente la política.

Una política impositiva a las dos energías que utilizan combustibles fósiles intensivamente: EPG y FPPC, provoca que la tasa de crecimiento del PIB sea inferior, respecto al escenario base, durante todo el periodo de análisis. Además, puede observarse en la misma Tabla 2 y en la Figura 2 que, conforme se incrementa la tasa de impuesto el impacto negativo sobre el PIB resulta mayor, por ejemplo, para el $5 \%$ de impuesto, la tasa de crecimiento es $0.6 \%$ menor y la diferencia se mantiene constante a lo largo del tiempo. Si la tasa de impuesto es de $10 \%$ y $15 \%$, el PIB decrece $1.1 \%$ y $1.6 \%$ respectivamente y este impacto también se mantiene en el tiempo. Se hace notar que a mayor impuesto mayor es la tasa de decrecimiento del PIB. 
Tabla 2: Resultados para una elasticidad de sustitución $\sigma=0.5$.

\begin{tabular}{l|cccccc}
\hline \hline \multirow{2}{*}{ Var. macroeconómicas } & \multicolumn{3}{|c}{$5 \%$} & \multicolumn{2}{c}{$10 \%$} & \multicolumn{2}{c}{$15 \%$} \\
\cline { 2 - 7 } & \multicolumn{3}{|c}{ Años } & \multicolumn{2}{c}{ Años } & \multicolumn{2}{c}{ Años } \\
& 15 & 25 & 15 & 25 & 15 & 25 \\
\hline PIB & -0.6 & -0.6 & -1.1 & -1.1 & -1.6 & -1.5 \\
Consumo & -0.6 & -0.6 & -1.2 & -1.2 & -1.7 & -1.7 \\
Inversión & -0.7 & -0.7 & -1.4 & -1.4 & -2 & -1.9 \\
Gasto público & -0.6 & -0.6 & -1.2 & -1.2 & -1.7 & -1.7 \\
Exportaciones & -0.6 & -0.6 & -1.2 & -1.1 & -1.7 & -1.6 \\
Importaciones & -0.8 & -0.8 & -1.6 & -1.6 & -2.3 & -2.3 \\
Utilidad & -0.03 & -0.05 & -0.1 & -0.1 & -0.1 & -0.15 \\
\hline \hline
\end{tabular}

La menor tasa de crecimiento del PIB se explica por la menor tasa de crecimiento de las variables agregadas, respecto a las que tendrían en el escenario base. En particular, el consumo, la inversión y el gasto público decrecen a una tasa muy similar y de manera constante para cada tasa impositiva, en cambio su comportamiento se asemeja al del PIB, a mayor tasa de impuesto, más grande es el impacto negativo sobre todas estas variables. En el caso de la demanda agregada, las tasas de decrecimiento son aproximadamente 0.6, 1.2 y 1.7 para los impuestos 5\%,10\% y $15 \%$ respectivamente. Esto se debe a que el mayor precio de los bienes energéticos de origen fósil se traslada al costo de producción de los bienes de consumo y de inversión que se producen internamente.

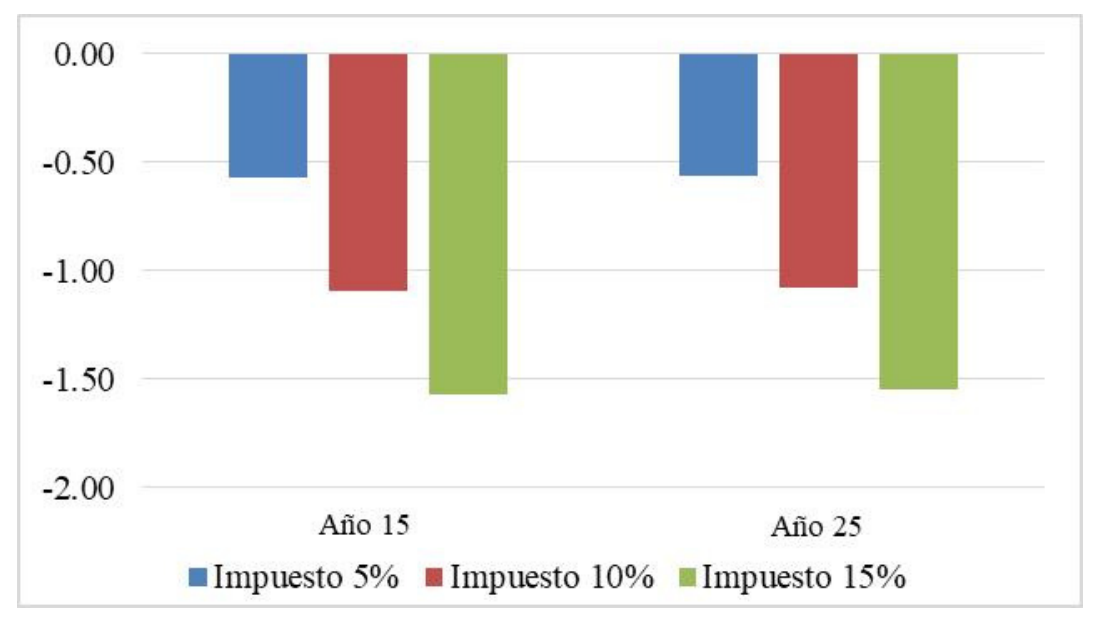

Figura 2: Impacto de los impuestos sobre el PIB, $\sigma=0.5$. 
En cuanto al comercio exterior, tanto las exportaciones como las importaciones crecen a menor tasa respecto al escenario base. Sin embargo, los efectos sobre la balanza comercial muestran una mejora en la misma respecto al escenario base, ya que hay una reducción de la demanda de los combustibles fósiles en el ámbito doméstico, mientras se supone que no hay cambios en el exterior.

El comportamiento de la balanza comercial se explica, por una parte, porque existe una pérdida de competitividad de los bienes nacionales con respecto al escenario base, debido al incremento de los precios relativos que ocasiona el impuesto sobre los insumos energéticos, lo que a su vez redunda en la disminución de las exportaciones, a lo largo de todo el periodo de análisis, a una tasa constante de aproximadamente $0.6,1.2$ y 1.7 para las tasas impositivas de 5,10 y 15 respectivamente. Las importaciones se reducen en mayor medida: $0.8 \%, 1.6 \% \mathrm{y}$ $2.3 \%$ para las tasas de 5,10 y 15 respectivamente, esto es en parte consecuencia del menor dinamismo de la demanda agregada y principalmente a la menor demanda de combustibles fósiles en toda la economía, respecto al escenario base, como se aprecia en la Tabla 2 .

\subsection{Efectos de los impuestos sobre las variables macroeconómicas para $\sigma=1.8$}

En el escenario en el que la economía es flexible, la política replica resultados similares: la tasa de crecimiento del PIB es inferior respecto al escenario base, durante todo el periodo de análisis, sin importar cuál sea la tasa impositiva con que se grave a las energías intensivas en el uso del carbón, como se aprecia en la Tabla 3.

Tabla 3: Resultados para una elasticidad de sustitución $\sigma=1.8$.

\begin{tabular}{l|cccccc}
\hline \hline \multirow{2}{*}{ Var. macroeconómicas } & \multicolumn{3}{|c}{$5 \%$} & \multicolumn{2}{c}{$10 \%$} & \multicolumn{2}{c}{$15 \%$} \\
\cline { 2 - 7 } & \multicolumn{2}{|c}{ Años } & \multicolumn{2}{c}{ Años } & \multicolumn{2}{c}{ Años } \\
& 15 & 25 & 15 & 25 & 15 & 25 \\
\hline PIB & -0.5 & -0.5 & -1 & -1 & -1.4 & -1.4 \\
Consumo & -0.6 & -0.6 & -1.2 & -1.2 & -1.6 & -1.6 \\
Inversión & -0.6 & -0.6 & -1.2 & -1.2 & -1.7 & -1.7 \\
Gasto público & -0.6 & -0.6 & -1.1 & -1.1 & -1.5 & -1.5 \\
Exportaciones & -0.6 & -0.6 & -1.1 & -1.1 & -1.6 & -1.5 \\
Importaciones & -0.9 & -0.9 & -1.7 & -1.7 & -2.4 & -2.3 \\
Utilidad & -0.04 & -0.04 & -0.1 & -0.09 & -0.12 & -0.14 \\
\hline \hline
\end{tabular}


También puede observarse en la Tabla 3 y en la Figura 3 , que mientras mayor sea la tasa de impuesto, más grande es el impacto negativo sobre el PIB. Así, para el $5 \%$ de impuesto, la tasa de crecimiento es menor en 0.5 respecto al escenario de referencia a lo largo del tiempo. Si la tasa de impuesto es de 10 y 15, el PIB decrece $1 \%$ y $1.4 \%$ respectivamente y este comportamiento también se mantiene en el tiempo.

Al igual que en el escenario anterior, una menor tasa de crecimiento del $\mathrm{PIB}$, respecto al escenario base, se explica por el decrecimiento de las variables agregadas. El consumo, la inversión y el gasto decrecen, a una tasa muy similar y de manera constante para cada tasa impositiva, en cambio, mientras mayor sea la tasa de impuesto, más grande es el impacto negativo sobre todas las variables que conforman la demanda agregada, aproximadamente $0.6 \%, 1.1 \%$ y $1.6 \%$ para las tasas de 5, 10 y 15 respectivamente. Esto se explica porque el mayor precio de los bienes EPG y FPPC se traslada a los costos de producción de los bienes de consumo.

Bajo este escenario, el costo de la política impositiva origina un decrecimiento del PIB menor en $0.1 \%$ para cada tasa impositiva respecto al escenario rígido. La diferencia en el comportamiento del PIB se debe a que, si bien existe una mayor facilidad para migrar insumos y factores hacia el sector productor de energías renovables, este sector representa apenas el 7.9\% de la producción total de energía en México [29]. Además, este bien energético es un insumo que únicamente demanda el sector productor de energía eléctrica.

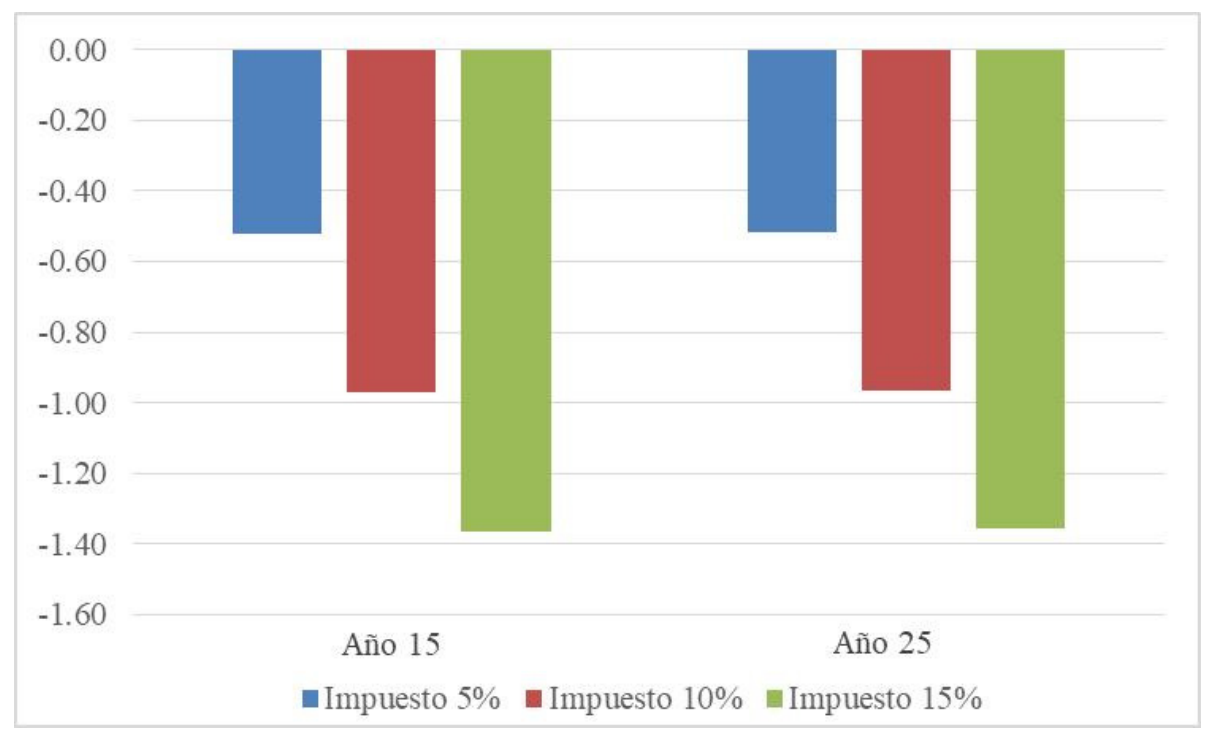

Figura 3: Impacto de los impuestos sobre el PIB, $\sigma=1.8$. 
En este escenario, al igual que en el rígido, el comercio internacional disminuye al reducirse tanto las exportaciones como las importaciones del país respecto al escenario inercial, pero la balanza comercial presenta una mejoría respecto al escenario base, debido a que el impuesto sobre los insumos energéticos ocasiona un incremento de los precios relativos, lo que a su vez, promueve la pérdida de competitividad de los bienes nacionales y redunda en la disminución de las exportaciones a lo largo de todo el periodo de análisis, a una tasa constante de aproximadamente $0.6,1.1$ y 1.6 para los impuestos de $5 \%, 10 \%$ y $15 \%$, respectivamente. Sin embargo, las importaciones se reducen en mayor medida: $0.9 \%, 1.7 \%$ y $2.3 \%$ para las tasas de 5,10 y 15 respectivamente, debido a la menor demanda de combustibles fósiles, respecto al escenario base, como se aprecia en la Tabla 3 .

Los resultados muestran que la reducción de gases de efecto invernadero a la atmósfera, tiene un costo macroeconómico frente a la opción de continuar con las emisiones sin buscar la sustitución de las energías contaminantes sin ninguna restricción.

\subsection{Efectos de los impuestos en el bienestar de la familia para $\sigma=0.5,1.8$}

Un aspecto relevante es el análisis del impacto de las políticas fiscales sobre el bienestar del consumidor. En las Tablas 2 y 3 , se muestran los datos de las variaciones porcentuales en la utilidad del consumidor representativo bajo los dos escenarios alternativos y su comparación con el escenario base.

Se observa que, en los dos escenarios alternativos, la variación del bienestar de los consumidores en términos de la utilidad es decreciente respecto al escenario base, además esta pérdida de bienestar se acentúa con el transcurso del tiempo y mientras mayor sea la tasa impositiva. En ambos escenarios, la utilidad total en el año 25, con la máxima tasa de impuesto, es aproximadamente $0.15 \%$ por debajo del escenario base, como se puede observar en la Figura 4 y en las Tablas $2 \mathrm{y} 3$.

Un resultado relevante hasta este punto es que la política fiscal simulada no permite obtener simultáneamente una mejora en el bienestar de los hogares y una reducción en la cantidad demandada de las energías intensivas en dióxido de carbono, las cuales se analizan a continuación. 


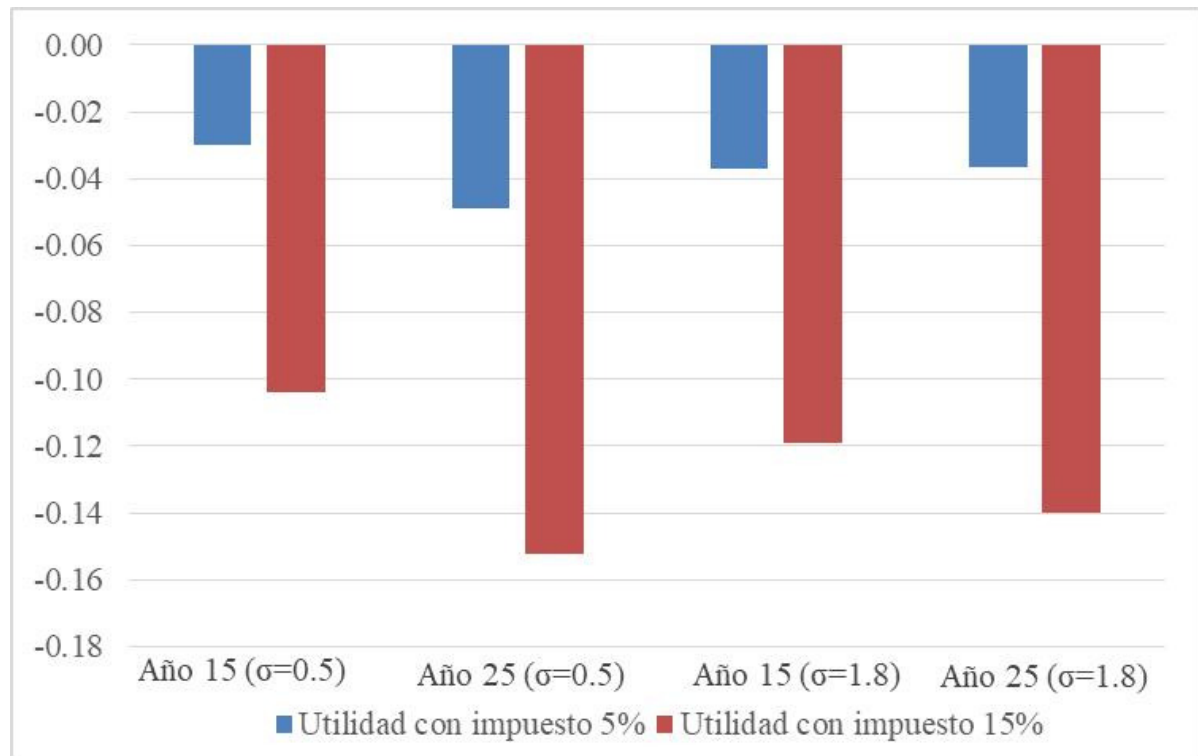

Figura 4: Bienestar de la familia, utilidad.

\subsection{Efectos de los impuestos a la demanda intermedia de bienes energéticos}

En esta sección se presentan las variaciones sobre las demandas de los bienes energéticos que se utilizan en esta economía como insumos o bienes intermedios. El incremento del precio relativo de cada uno de los bienes, por la carga impositiva, induce una disminución en su cantidad demandada. También, se evalúa cómo se modifican las cantidades demandadas del otro bien energético tradicional, GEE, cuya producción requiere de REN.

En general, el mecanismo de transmisión y los efectos finales suceden de acuerdo con los supuestos de anidamiento y flexibilidad de sustitución entre los bienes energéticos que conforman al bien energético agregado, el resto de los insumos intermedios y el valor añadido. Se debe enfatizar que los resultados obtenidos dependen del valor que adopte la elasticidad de sustitución de los bienes energéticos en ambos escenarios, es decir, el rígido $(\sigma=0.5)$ y el flexible $(\sigma=1.8)$. 


\subsubsection{Efectos de los impuestos a la demanda intermedia de bienes energéticos $\sigma=0.5$}

En la Tabla 4 y en la Figura 5 se exhiben los efectos sobre la cantidad demandada de las energías que tiene la política de gravar de forma simultánea, con un impuesto de 5\%, 10\% y 15\% a los bienes EPG y FPPC, en el escenario rígido.

Tabla 4: Porcentaje de variación de la demanda intermedia de bienes energéticos $(\sigma=0.5)$.

\begin{tabular}{l|cccccc}
\hline \hline \multirow{3}{*}{ Demanda de energéticos } & \multicolumn{3}{|c}{$5 \%$} & \multicolumn{2}{c}{$10 \%$} & \multicolumn{2}{c}{$15 \%$} \\
\cline { 2 - 8 } & \multicolumn{2}{|c}{ Años } & \multicolumn{2}{c}{ Años } & \multicolumn{2}{c}{ Años } \\
& 15 & 25 & 15 & 25 & 15 & 25 \\
\hline GEE & -2 & -2 & -3.9 & -3.9 & -5.7 & -5.7 \\
EPG & -4.4 & -4.4 & -8.5 & -8.5 & -12.2 & -12.3 \\
FPPC & -3.7 & -3.7 & -7.2 & -7.2 & -10.4 & -10.4 \\
REN & -2.1 & -2.1 & -4 & -4.1 & -5.9 & -5.9 \\
\hline \hline
\end{tabular}

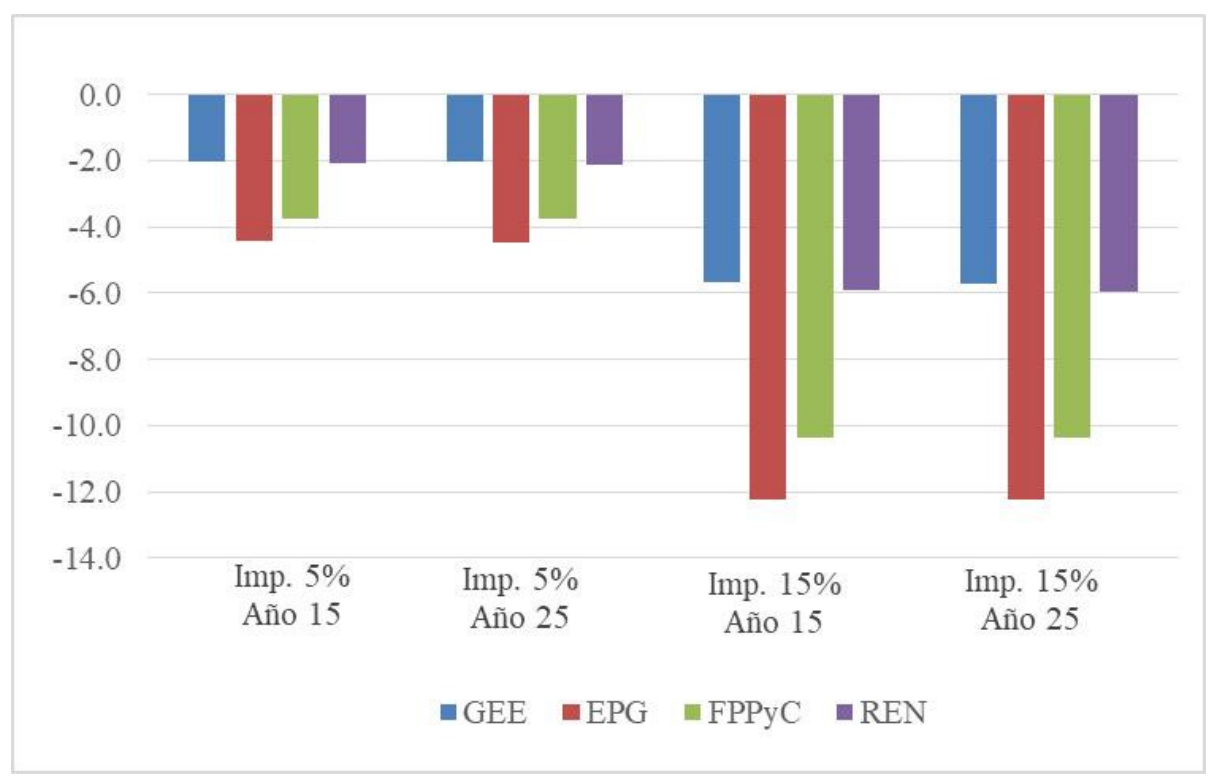

Figura 5: Efectos de los impuestos sobre las demandas de energías, $\sigma=0.5$. 
Se observa en general, que las cantidades demandadas de cada una de las energías consideradas, decrece respecto al escenario base o inercial, incluyendo la del bien REN y la GEE, aunque estas lo hacen en menor medida. Por ejemplo, para la tasa impositiva de $5 \%$, la cantidad demandada de estos bienes disminuye $2 \%$, mientras que las correspondientes a los bienes energéticos intensivos en carbono, EPG y FPPC, decrecen aproximadamente $4 \%$; cabe señalar que estas tasas se mantienen casi constantes a lo largo del horizonte de planeación. Cuando la tasa de impuesto se incrementa, se acentúa la tendencia decreciente de las demandas de energía, así, para una tasa impositiva de 15, las demandas de EPG y FPPC, disminuyen aproximadamente $12 \%$ y $10 \%$, por su parte, la demanda de los bienes GEE y REN decrece, respecto al escenario base, casi el $6 \%$, este comportamiento se mantiene durante los 25 años.

\subsubsection{Efectos de los impuestos a la demanda intermedia de bienes energéticos $\sigma=1.8$}

En el escenario con una elasticidad de sustitución flexible, esta tendencia se revierte para los insumos energéticos GEE y REN, cuyas demandas crecen continuamente y en mayor medida cuando se incrementa el impuesto, como se puede apreciar en la Tabla 5 y en la Figura 6. Así, para un impuesto de $5 \%$, ambas demandas crecen casi $1 \%$ y $4 \%$ respectivamente, manteniendo estos valores hasta culminar el año 25; en cuanto a las demandas de las energías EPG y FPPC, con esta misma tasa de impuesto, decrecen aproximadamente $5 \%$ y $6 \%$ manteniéndose así hasta el año 25.

Tabla 5: Porcentaje de variación de la demanda intermedia de bienes energéticos $(\sigma=1.8)$.

\begin{tabular}{l|cccccc}
\hline \hline \multirow{2}{*}{ Demanda de energéticos } & \multicolumn{2}{|c}{$5 \%$} & \multicolumn{2}{c}{$10 \%$} & \multicolumn{2}{c}{$15 \%$} \\
\cline { 2 - 7 } & \multicolumn{2}{|c}{ Años } & \multicolumn{2}{c}{ Años } & \multicolumn{2}{c}{ Años } \\
GEE & 0.7 & 25 & 15 & 25 & 15 & 25 \\
EPG & -5.3 & -5.3 & -10 & -10.1 & -14.4 & -14.4 \\
FPPC & -5.9 & -5.9 & -11 & -11.3 & -16.1 & -16.1 \\
REN & 3.5 & 3.5 & 6.9 & 6.8 & 10.2 & 10.1 \\
\hline \hline
\end{tabular}




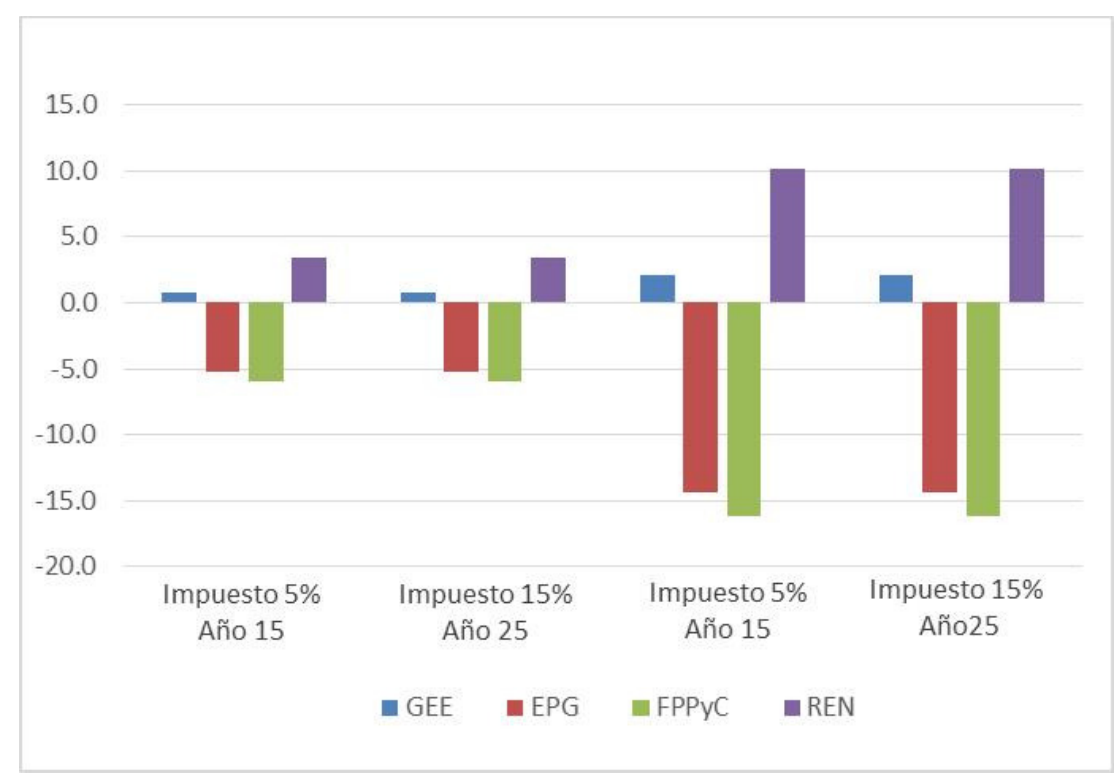

Figura 6: Efectos de los impuestos sobre las demandas de energías, $\sigma=1.8$.

A medida que la tasa impositiva aumenta, nuevamente se acentúa la tendencia presentada y las demandas de GEE y REN se incrementan respecto al escenario base o inercial, en $2 \%$ y $10 \%$ respectivamente, manteniéndose así hasta el año 25. Esto ocurre así, debido a que en este escenario se pueden sustituir las energías contaminantes que han sido gravadas por el bien GEE, de esta forma, al aumentar la demanda de GEE se favorece al crecimiento de la demanda de la energía renovable, por ser el único sector energético que la utiliza como insumo. Por otra parte, las energías tradicionales EPG y FPPC continúan su decrecimiento, de manera natural, conforme aumenta el nivel de impuesto y terminan con tasas de $-14 \%$ y $-16 \%$ respectivamente, hasta el año 25 .

Por lo tanto, aplicar impuestos al uso de las energías fósiles, resulta una política efectiva únicamente en el escenario flexible, si el objetivo consiste en disminuir el uso de las energías contaminantes EPG y FPPC, y sustituirlas por REN, al reducir la contaminación atmosférica debida a la producción y consumo de combustibles fósiles. La efectividad de la política aumenta a medida que la economía tenga la capacidad de sustituir con mayor flexibilidad los insumos energéticos en el proceso productivo. Sin embargo, se recomienda evaluar con precisión el efecto distorsionante del impuesto y comparar sus costos en crecimiento económico y bienestar con el beneficio obtenido por la reducción en la emisión de contaminantes. 


\section{Conclusiones}

La interpretación de los resultados muestra que la eficacia de una política ambiental que favorece la sustitución de las energías fósiles por energías renovables, depende de la elasticidad de sustitución que exista en la economía. Si se presentan dificultades técnicas para sustituir los insumos energéticos, entonces la política fiscal es parcialmente efectiva, ya que se reducen las demandas de todas las energías, sean o no contaminantes y el costo en la producción es mayor que en el escenario de flexibilidad.

Los resultados también evidencian que no es posible llevar a cabo una política medioambiental que ofrezca a la economía mexicana una mejora en el bienestar social y en la reducción de las emisiones de gases de efecto invernadero, a través de menor consumo de los insumos energéticos contaminantes. En los dos escenarios se constata que la variación del bienestar de la familia en términos de la utilidad es decreciente respecto al escenario base, al igual que en la producción, la pérdida en bienestar resulta superior en el caso de rigidez en la sustitución. Asimismo, los resultados muestran que bajo un mismo escenario, los costos en la producción y el bienestar son mayores mientras las tasas de impuestos sean más altas.

Ya que en el escenario de rigidez es mayor el costo en el PIB y en el bienestar social, el diseño de una política impositiva que tenga un menor impacto negativo sobre el crecimiento de la producción, debe tomar en cuenta el parámetro de la elasticidad de sustitución existente en la economía.

En lo referente a la sustitución de insumos, la efectividad de la política fiscal aumenta, a medida que exista una mayor elasticidad de sustitución técnica entre las energías. Por lo tanto, es necesario promover el cambio tecnológico que acompañe a la política propuesta, a fin de tener una mayor posibilidad de sustituir las energías tradicionales por energías renovables.

Es necesario contar con estudios donde se estime el valor de la elasticidad para la economía mexicana contemporánea, así como las políticas que podrían utilizarse para inducir un cambio tecnológico hacia un mayor uso de las energías renovables.

\section{Agradecimientos}

Los autores agradecen el apoyo brindado por la Universidad Autónoma Metropolitana, Unidad Iztapalapa, en particular a los Departamentos de Economía, Ingeniería Eléctrica y Matemáticas. 


\section{Referencias}

[1] E. Aguayo, J. Chapa, N. Ramírez, E. Rangel, Análisis de la generación y redistribución del ingreso en México a través de una matriz de contabilidad social, Estudios Económicos (2009), 225-311.

[2] C. Böhringer, A. Löschel, Promoting renewable energy in Europe: a hybrid computable general equilibrium approach, The Energy Journal 27 (2006), no. $1,135-150$.

[3] R. Boyd, M. Ibarrarán, Costs of compliance with the Kyoto protocol: a developing country perspective, Energy Economics 24 (2002), no.1, 2139.

[4] H. Bravo, J. Castro, M. Gutiérrez, E. Omaña, Evaluación de una política de sustitución de energías fósiles para reducir las emisiones de carbono, El Trimestre Económico 84 (2017), no. 333, 137-164.

[5] P. Dixon, S. Osborne, M. T. Rimmer, The economy-wide effects in the United States of replacing crude petroleum with biomass, Energy and Environment 6 (2007), no. 6, 709-722.

[6] V. Ginsburgh, M. Keyzer, The structure of applied general equilibrium models, The MIT Press, Cambridge, 1997.

[7] M. González-Eguino, Los costes de mitigar el cambio climático: un análisis dinámico de equilibrio general aplicado, Revista de Economía Aplicada XIX (2011), no. 56, 89-121.

[8] L. Goulder, Enviromental taxation and the double dividend: a reader's guide, International Tax and Public Finance 2 (1995), no. 2, 157-183.

[9] D. Greenaway, S. J. Leybourne, G. V. Reed, J. Whalley,Applied general equilibrium modelling: applications, limits and future development, HMSO, Londres, 1993.

[10] Z. Guo, X. Zhang, Y. Zheng, R. Rao, Exploring the impacts of a carbon tax on the chinese economy using a CGE model with a detailed disaggregation of energy sectors, Energy Economics 45 (2014), no. C, 455-462.

[11] B. Heer, A. Maussner, Dynamical general equilibrium modeling: Computational Methods and Applications, 2nd ed., Springer Verlag, Heilderberg, 2009. 
[12] J. Jensen, T. Rasmussen, Allocation of $\mathrm{CO}_{2}$ emissions permits: a general equilibrium analysis of policy instruments, Journal of Environmental Economics and Management 40 (2000), no. 2, 111-136.

[13] D. W. Jorgenson, R. Goettle, M. Ho, P. Wilcoxen, Double dividend: environmental taxes and fiscal reform in the United States, MIT Press, Cambridge, 2013.

[14] R. Kaufmann, Limits on the economic effectiveness of a carbon tax, The Energy Journal 12 (1991), no. 4, 139-144.

[15] B. Kretschmer, S. Peterson, Integrating bioenergy into computable general equilibrium models - a survey, Energy Economics 32 (2010), no. 3, 673-686.

[16] G. Landa, F. Reynes I. Islas, F. Bellocq, F. Grazi, Towards a low carbon growth in Mexico: is a double dividend possible? A dynamic general equilibrium assessment, Energy Policy 96 (2016), 314-327.

[17] OECD/IEA, Energy policies beyond IEA countries: México 2017, https://webstore.iea.org/ energy-policies-beyond-iea-countries-mexico-2017, consulted on 04-Feb-2019, 15:30 p.m.(2017)

[18] OECD/IEA, Mexico energy outlook, world energy outlook special report, https://www.iea.org/publications/ freepublications/publication/MexicoEnergyOutlook. pdf, consulted on 04-Feb-2019, 15:34 p.m.

[19] S. Paltsev, Moving from static to dynamical general equilibrium economic models (Notes for beginner in MPSGE), Joint Program on the Science and Policy of Global Change, 2004.

[20] S. Paltsev, J. Reilly, H. Jacoby, R. Eckaus, J. McFarland, M. Saro?m, M. Asadoorian, M. Babiker, The MIT emissions prediction and policy analysis (EPPA) model: version 4, MIT Joint Program on the Science and Policy of Global Change, Report. 125, Cambridge, 2005.

[21] C. Papageorgiou, M. Saam, P. Schulte, Elasticity of substitution between clean and dirty energy inputs: a macroeconomic perspective, ZEW- Centre for European Economic Research, Discussion Paper (2013), no. 13-087. 
[22] K. Pearson, B. R. Parmenter, A. A. Powell, P. J. Wilcoxen, P. B. Dixon, Notes and problems in applied general equilibrium economics, 1st ed., Elsevier, 1992.

[23] S. Rausch, M. Mowers, Distributional and efficiency impacts of clean and renewable energy standards for electricity, Resource and Energy Economics 36 (2014), no. 2, 556-585.

[24] J. Romero, Energía, emisiones y precios relativos (Antonio Yúnez-Naude, ed.), Medio Ambiente: Problemas y Soluciones, Colegio de México, 1994.

[25] T. Rutherford, Applied general equilibrium modeling with MPSGE as a GAMS subsystem: an overview of the modeling framework and syntax, preprint, 1995.

[26] T. Rutherford, Extension of GAMS for complementary problems arising in applied economic analysis, Journal of Economic Dynamics and Control 19 (1995), no. 8, 1299-1324.

[27] T. Rutherford, Dynamic general equilibrium with GAMS/MPSGE, Lectures Notes Prepared for the UNSW Workshop, 2004.

[28] X. Sala-i-Martin, Apuntes de crecimiento económico, 2nd ed., Antoni Bosch Editor S.A., Madrid, 2000.

[29] SENER, Balance nacional de energía 2015, https://www.gob. $\mathrm{mx} / \mathrm{cms} / \mathrm{uploads} /$ attachment/file/248570/Balance_ Nacional_de_Energ_a_2015_2_.pdf

[30] SENER/CONUEE, Estrategia de transición para promover el uso de tecnologías y combustibles más limpios, https://wWw.gob.mx/cms/ uploads/attachment/file/182202/20161110_1300h_ Estrategia_CCTE-1.pdf

[31] G. Solveig, H. Vennemo, T. Johnsen, Stabilization of emissions of $\mathrm{CO}_{2}:$ a computable general equilibrium assessment, Scandinavian Journal of Economics 94 (1992), no. 1, 53-69.

[32] L. Tang, J. Shi, L. Yu, Q. Bao, Economic and environmental influences of coal resource tax in China: a dynamic computable general equilibrium approach, Resources, Conservation and Recycling 117 (2017), 34-44. 
[33] G. Timilsina, R. Shrestha, General equilibrium analysis of economic and environmental effects of carbon tax in a developing country: case of Thailand, Environmental Economics and Policy Studies 5 (2002), no. 3, 179-211.

[34] UNEP/ONU, Resumen de las evaluaciones regionales del sexto informe sobre las perspectivas del medio ambiente mundial: resultados principales y mensajes políticos, https://wedocs.unep.org/ bitstream/handle/20.500.11822/7688/-Resumen_de_ las_evaluaciones_regionales_del_sexto_informe_ sobre_las_perspectivaas_del_medio_ambiente_ mundial_GEO-6_Resultados_principales_y_mensajes_ .pdf? sequence $=3 \&$ isAllowed $=y$

[35] Y. Yuan, Y. Lu, Dynamic general equilibrium analysis on the impacts of carbon tax on Chinese economy, 2011 2nd International Conference on Artificial Intelligence, Management Science and Electronic Commerce, Deng Feng, China, 2011.

\section{Anexo}

En esta sección se describe la Matriz de Contabilidad Social.

Tabla 6: Primera parte de la SAM. Representa el comportamiento de los seis sectores productivos y de algunos agentes.

\begin{tabular}{c|cccccc}
\hline \hline Actividad & SPR & ISE & GEE & EPG & FPP & REN \\
\hline SPR & 65937 & 391993 & 127 & 1982 & 934 & \\
ISE & 111204 & 4073079 & 77206 & 7570 & 14155 & \\
GEE & 7196 & 133118 & 29846 & 182 & 720 & \\
EPG & 0 & 934 & 45435 & 0 & 0 & \\
FPP & 2550 & 139878 & 674 & 0 & 0 & \\
REN & & & 11539 & & & \\
FACTR & 53059 & 1780681 & 35300 & 11994 & 7045 & 347 \\
FACAP & 233068 & 4231429 & 36259 & 57518 & 9272 & 8067 \\
FAM & & & & & & \\
GOB & 56812 & 806344 & -1578 & 69483 & 1942 & \\
AHO & & & & & & \\
SE & 126139 & 2244061 & 27881 & 0 & 214142 & \\
TOT & 655965 & 13801517 & 262689 & 148729 & 248210 & 11539 \\
\hline \hline
\end{tabular}


Tabla 7: Segunda parte de la SAM. Se presentan los ingresos de la familia por la renta de los factores y sus egresos.

\begin{tabular}{c|ccc}
\hline \hline Actividad & FACTR & FACAP & FAM \\
\hline SPR & & & 122348 \\
ISE & & & 4972008 \\
GEE & & & 80369 \\
EPG & & & 0 \\
FPP & & & 78687 \\
REN & & & 0 \\
FACTR & & & \\
FACAP & & & \\
FAM & 2166581 & 4622565 & \\
GOB & & & 368114 \\
AHO & & & 1564661 \\
SE & & 80483 & \\
TOT & 2166581 & 4703048 & 12256757 \\
\hline \hline
\end{tabular}

Tabla 8: Tercera parte de la SAM. Se contabilizan claramente los egresos del gobierno, de las inversiones y del sector externo.

\begin{tabular}{c|ccc}
\hline Actividad & GOB & AHO & SE \\
\hline SPR & 2566 & 6239 & 63839 \\
ISE & 592323 & 1689683 & 2264289 \\
GEE & 9383 & 0 & 1875 \\
EPG & 0 & 124 & 102236 \\
FPP & 769 & 3200 & 22452 \\
REN & 0 & 0 & 0 \\
FACTR & 275030 & & 0 \\
FACAP & 127435 & & 0 \\
FAM & 181031 & & 216010 \\
GOB & & & \\
AHO & 60247 & & 77071 \\
SE & 52333 & 2733 & 0 \\
TOT & 1301117 & 1701979 & 2747772 \\
\hline \hline
\end{tabular}


